\title{
Adipocytokines: unravelling the missing link in diabetes and metabolic syndrome
}

\begin{abstract}
Type 2 diabetes mellitus (T2DM) and metabolic syndrome (MetS) is a major growing public health concern worldwide affecting approximately over 200 million individuals. It is on track to become one of the major global public health challenges of the 21 st century. Prevalence of diabetes worldwide was expected to be $2.8 \%$ in 2000 and $4.4 \%$ in 20301 and $80 \%$ of these cases were related to obesity. India has the dubious distinction of being known as the "Diabetic Capital of the World" as the diabetic population is increasing in a geometric progression. There is an urgent need to understand the underlying etiology and develop primary prevention strategies to control this epidemic.2,3 With advancement in the technologies for early detection, intervention, and prompt treatment of diseases, there is a growing scope for finding the most specific and sensitive biomarkers.
\end{abstract}

Volume 2 Issue 3 - 2017

\author{
Sabhiya Majid, Rabia Farooq, Showkat Ahmad \\ Bhat \\ Department of Biochemistry, University of Kashmir, India
}

Correspondence: Sabhiya Majid, Department of Biochemistry, University of Kashmir, India, Tel 978735 II,

Email sabumajid@yahoo.com

Received: October 25, 2017 | Published: December 14, 2017

\section{Introduction}

Adipose tissue - composed of adipocytes, fibroblasts, immune cells, and various other cell types, once known as passive reservoir is now considered an endocrine organ secreting bioactive molecules including hormones now termed adipokines with various known and unknown endocrine functions in addition to regulating fat mass and nutrient homeostasis. Due to the dramatic rise in obesity and its metabolic sequelae during the past decades, adipose tissue gained tremendous scientific interest. Adipose tissue secretes variety of products known as 'adipokines', including leptin, adiponectin, resistin and visfatin, as well as cytokines and chemokines such as tumor necrosis factor- Alpha (TNF- $\alpha$ ), interleukin- 6 and monocyte chemoattractant protein-1. These adipokines helps in the regulation of hemostasis, blood pressure, lipid and glucose metabolism, inflammation, and atherosclerosis. The release of these cytokines causes a chronic sub inflammatory state that could play a central role in the development of insulin resistance and type 2 diabetes, and the increased risk of cardiovascular disease associated with obesity, together referred as metabolic syndrome. ${ }^{4}$ This chronic low-grade inflammation causes increase in macrophage infiltration, leads to increased adipocyte secretion of pro-inflammatory molecules such as resistin, tumor necrosis factor- Alpha, and interleukin-6(IL-6). ${ }^{4}$ These adipocytokines can act as key regulators of response to insulin in peripheral tissues. ${ }^{5}$ Their role in causing insulin resistance via various mechanisms involving the flux of in pro-oxidant and antioxidant state has been seen. ${ }^{6,7}$ Adipokines have a great potential for clinical use as potential therapeutics for obesity, obesity related metabolic, cardiovascular and other diseases. ${ }^{8}$ Several extracellular factors cause obesity, related adipocyte metabolism and macrophage infiltration. Interestingly, recent research provides increasing evidence of the importance of regulating adipocyte function, adipose tissue metabolism and inflammation. In this mini review, we will briefly highlight roles of various adipokines (adiponectin, leptin, resistin, TNF- $\alpha$, and IL-6) in regulating insulin sensitivity and resistance.

\section{Main body}

\section{Adiponectin and Diabetes/MetS}

Adiponectin is an adipocyte-specific hormone known to be involved in a variety of metabolic, anti-inflammatory, and vasoprotective functions. Increase in body mass index leads to insulin resistance and type 2 diabetes. Hypoadiponectinemia correlates with the development of insulin resistance and type 2 diabetes. ${ }^{8}{ }^{89}$ Adiponectin promotes an insulin-sensitizing effect. ${ }^{10}$ Adiponectin could suppress glucose production and increase insulin sensitivity. ${ }^{11}$ The availability of adiponectin might reverse insulin resistance and therefore lead to decrease the risk of diabetes.

\section{Leptin and Diabetes/MetS}

Genetic defects in anorexigenic pathway can cause obesity, such as mutations in the melanocortin- 4 or leptin receptors. ${ }^{12}$ Its levels are estimated to be higher in persons with diabetes mellitus. ${ }^{13}$ Diet and leptin treatment is explored as a method of diabetes control. Obesity is not only influenced by lack of leptin but also leptin resistance. Leptin has been proven to increase with increasing adiposity among humans and rodents. ${ }^{14}$ Leptin polymorphism has a role in hypertension. ${ }^{15}$

\section{Resistin and Diabetes/MetS}

Resistin was latest adipokine to be discovered in the year 2001, resistin (RETN) genes helps in adipocyte differentiation, these were down regulated in mature adipocytes during exposure to Thiazolidinediones (TZD). Resistin was shown to be highly expressed among obese cases. ${ }^{16}$ Exposure to higher glucose concentrations significantly increased resistin expression in 3T3-L1 adipocytes, while treatment with insulin reduced it. ${ }^{17}$

\section{IL-6 and Diabetes/MetS}

IL-6 is a multifunctional cytokine that plays numerous roles in addition to controlling immune cell function, such as behaving as a hepatocyte-stimulating factor and a growth factor for metastatic cells. $^{18}$ T2DM shows elevated circulating levels of Interleukins and IL-6 is one among them. ${ }^{19}$ IL- 6 has been shown to activate SOCS-1 and -3 proteins in the liver, thus accompanying insulin resistance. ${ }^{20}$

\section{Conclusion}

Researches provide strong evidence that adipose tissue plays important roles as an endocrine organ. This vast organ is capable of regulating many physiological processes by the secretion of adipokines 
like adiponectin, leptin, resistin, TNF- $\alpha$, and IL-6. Adiponectin acts as an insulin-sensitizer and a target of anti-diabetic drugs, leptin has been shown to reverse insulin resistance. Resistin, has been shown to target key nodes of the insulin signaling pathway to induce insulin desensitization. IL-6, a multifunctional cytokine, has been shown to alter insulin sensitivity both in vivo and in vitro studies. While further research is still required, to investigate the cellular level effect that mechanical loading environment has on the adipokine secretory response of adipocytes in an effort to further illuminate the role of adipokines in insulin signaling. The broader implications of such research include a more advanced understanding of type-2 DM and revealing potential novel targets for anti-diabetic drugs.

\section{Acknowledgements}

None.

\section{Conflict of interest}

The author declares no conflict of interest.

\section{References}

1. Wild S, Roglic G, Green A, et al. Global prevalence of diabetes: estimates for the year 2000 and projections for 2030. Diabetes Care. 2004;27(5):1047-1053.

2. $\mathrm{Xu} \mathrm{J}, \mathrm{Xu} \mathrm{W}$, Yao H, et al. Associations of serum and urinary magnesium with the pre-diabetes, Diabetes and diabetic complications in the chinese northeast population. PLOS ONE. 2013;8(2):e56750.

3. Salem M, Kholoussi S, Kholoussi N, et al. Malondialdehyde and trace element levels in patients with type 2 diabetes mellitus. Arch Hellen Med. 2011;28(1):83-88.

4. Jung UJ, Choi M. Obesity and its metabolic complications: The role of adipokines and the relationship between obesity, inflammation, insulin resistance, dyslipidemia and nonalcoholic fatty liver disease. Int $\mathrm{J} \mathrm{Mol}$ Sci. 2014;15(4):6184-6223

5. Moonishaa TM, Nanda SK, Shamraj M, et al. Evaluation of leptin as a marker of insulin resistance in type 2 diabetes mellitus. Int J Appl Basic Med Res. 2017;7(3):176-180.

6. Irfan M, Rabia F, Rawoof M, et al. Estimation of lipid peroxidation and lipid profile levels in type II diabetes mellitus patients of Kashmir valley. IJALS. 2015;8(4):448-55.

7. Rashid T, Bhat SA, Wani MU, et al. The lipid peroxidation and antioxidant status of type 2 diabetic patients in Kashmir. Int J Diabetes Dev countries. 2015;35(4):476-481.
8. Blüher M, Mantzoros CS. From leptin to other adipokines in health and disease: Facts and expectations at the beginning of the $21^{\text {st }}$ century. Metab Clin Exp. 2015;64(1):131-145.

9. Lu HL, Wang HW, Wen Y, et al. Roles of adipocyte derived hormone adiponectin and resistin in insulin resistance of type 2 diabetes. World $J$ Gastroenterol. 2006;12(11):1747-1751.

10. Rabia F, Shajrul A, Rawoof M, et al. Type 2 diabetes and metabolic syndrome-adipokine levels and effect of drugs. Gynecological Endocrinology. 2016;33(1):75-78.

11. Berg AH, Combs TP, Du X, et al. The adipocyte-secreted protein Acrp30 enhances hepatic insulin action. Nat Med. 2001;7(8):947-953

12. Vrachnis N, Belitsos P, Sifakis S, et al. Role of adipokines and other inflammatory mediators in gestational diabetes mellitus and previous gestational diabetes mellitus. 2012;32(10):607-616.

13. Marzo VD, Goparaju SK, Wang L, et al. Leptin-regulated endocannabinoids are involved in maintaining food intake. Nature. 2001;410:822-825.

14. Maffei M, Halaas J, Ravussin E, et al. Leptin levels in human and rodent: measurement of plasma leptin and ob RNA in obese and weight-reduced subjects. Nat Med. 1995;1(11):1155-1161

15. Qulsum A, Akbar M, Ruhi A, et al. Polymorphisms in the 3'UTR of the human leptin gene and heir role in hypertension. Mol Med Reports. 20121;5(4):1058-1062.

16. Ternan PG, Ternan CL, Chetty $\mathrm{R}$, et al. Increased resistin gene and protein expression in human abdominal adipose tissue. J Clin Endocrinol Metab. 2002;87(5):2407.

17. Shojima N, Sakoda H, Ogihara $\mathrm{T}$, et al. Humoral regulation of resistin expression in 3T3-L1 and mouse adipose cells. Diabetes. 2002;51(6):1737-1744

18. Kishimoto T, Akira S, Taga T. Interleukin-6 and its receptor: A paradigm for cytokines. Science. 1992;258(5082):593-597.

19. Vozarova B, Weyer C, Hanson K, et al. Circulating interleukin-6 in relation to adiposity, insulin action, and insulin secretion. Obes Res. 2001;9(7):414-417

20. Senn JJ, Klover PJ, Nowak IA, Zimmers TA, Koniaris LG, et al. (2003) Suppressor of cytokine signaling-3 (SOCS-3), a potential mediator of interleukin-6-dependent insulin resistance in hepatocytes. J Biol Chem. 278(16): 13740-13746. 